\title{
El reto del maíz en México frente al cambio climático
}

\author{
Guillermo N. Murray Tortarolo \\ Víctor J. Jaramillo
}

\section{Resumen}

El maíz es la base de la alimentación de la gran mayoría de los mexicanos. Lo comemos en tortillas, pozole, tamales, tostadas y hasta en la sopa. No obstante, es aun de mayor importancia para los campesinos de subsistencia que dependen directamente de él para vivir. El maíz es, a su vez, altamente dependiente de la variabilidad interanual de la lluvia, y la sequía representa un riesgo importante para su producción a nivel nacional. Los escenarios futuros muestran una reducción de 10\% en la producción de maíz en México y hasta de 30\% en ciertas regiones. Esto representa una potencial crisis social y ambiental en las próximas décadas. Es necesario proponer estrategias para enfrentarlas y muchas de ellas están en tus manos.

Palabras clave: sequía, agricultura de subsistencia, maíz de temporal, calentamiento global, agrotecnología.

\section{Maize and climate change in Mexico}

\begin{abstract}
Maize is the cornerstone of the nutrition for a great number of mexicans. We eat it in all sorts of presentations: tortillas, pozole, tamales, and even in soups. However, it is of major importance for the small-scale farmers of the country, who depend directly on its yields for their survival. This crop is particularly sensitive to rainfall variability and climate change represents a high risk for its production nationwide. Future scenarios predict a reduction of up to 10\% in maize production in Mexico and up to 30\% regionally within the country. This represents potential social and environmental crises. We need to come up with strategies to face them, many of which are in your hands.
\end{abstract}

Key words: drought, subsistence agriculture, rainfed maize, global warming, agrotechnologies. 


\section{Guillermo N. Murray-Tortarolo}

Es Investigador Cátedra CONACyT comisionado al Instituto de Investigaciones en Ecosistemas y Sustentabilidad. Universidad Nacional Autónoma de México. Mi tema principal de trabajo es la investigación socio-ecológica para el manejo sustentable de ecosistemas a nivel nacional y global. Me interesa entender los impactos del cambio y la variación climática sobre distintos procesos ecológicos y agronómicos de México y el mundo. También tengo un profundo interés y pasión por la divulgación de la ciencia y por la enseñanza de la misma.

Víctor J. Jaramillo

luque@iies.unam.mx

Instituto de Investigaciones en Ecosistemas y Sustentabilidad. Universidad Nacional Autónoma de México. Mi línea principal de investigación es la biogeoquímica de ecosistemas tropicales, principalmente del carbono, nitrógeno y fósforo, con un énfasis en los ecosistemas tropicales estacionalmente secos. Me interesa caracterizar y entender procesos vinculados con los nutrientes en los flujos hidrológicos, en las plantas y en el suelo, con estudios de corto y largo plazo. Mis proyectos se han enfocado tanto en la selva no perturbada, como en el impacto de la transformación de la misma con fines agropecuarios a través de la roza, tumba y quema. He sido un activo participante en la enseñanza de la ecología en licenciatura y posgrado y en programas de investigación de índole global.

\section{El maíz que corre en nuestra sangre}

Los seres humanos fuimos hechos de maíz por los progenitores, creadores y formadores; de mazorcas nuestro esqueleto y de masa de maíz nuestra sangre y nuestra carne. Así lo dice el Popol-Vuh y así lo creyeron los habitantes ancestrales de nuestro país por más de mil años. Sí, es cierto que la ciencia moderna ha revelado que en realidad venimos de un largo proceso de evolución, pero de alguna manera sí estamos hechos de maíz, pues es la pieza fundamental de la dieta de la gran mayoría de los mexicanos; al final, somos lo que comemos.

Para darnos una idea, en México una persona consume, en promedio, entre 100 y 200 kilogramos de maíz por año en sus diferentes presentaciones. Solamente en forma de tortilla se estima que cada uno de nosotros come entre 50 y 90 kilogramos por año y el resto en sus diferentes manifestaciones: cereales, pozole, tamales, atole, tostadas, palomitas, chilaquiles, enchiladas, gorditas, elotes... en fin, un sinnúmero de productos que nos mantienen gorditos y felices. Somos, sin duda alguna, un país taquero y las cifras muestran que lejos de alejarnos de dichos productos, cada día los comemos más. 
La creación del Popol-vuh, norte, sur y centro. Autor: Cuilomerto. https://commons.wikimedia.org/ wiki/File:Norte,_Sur_y_Centro.png.

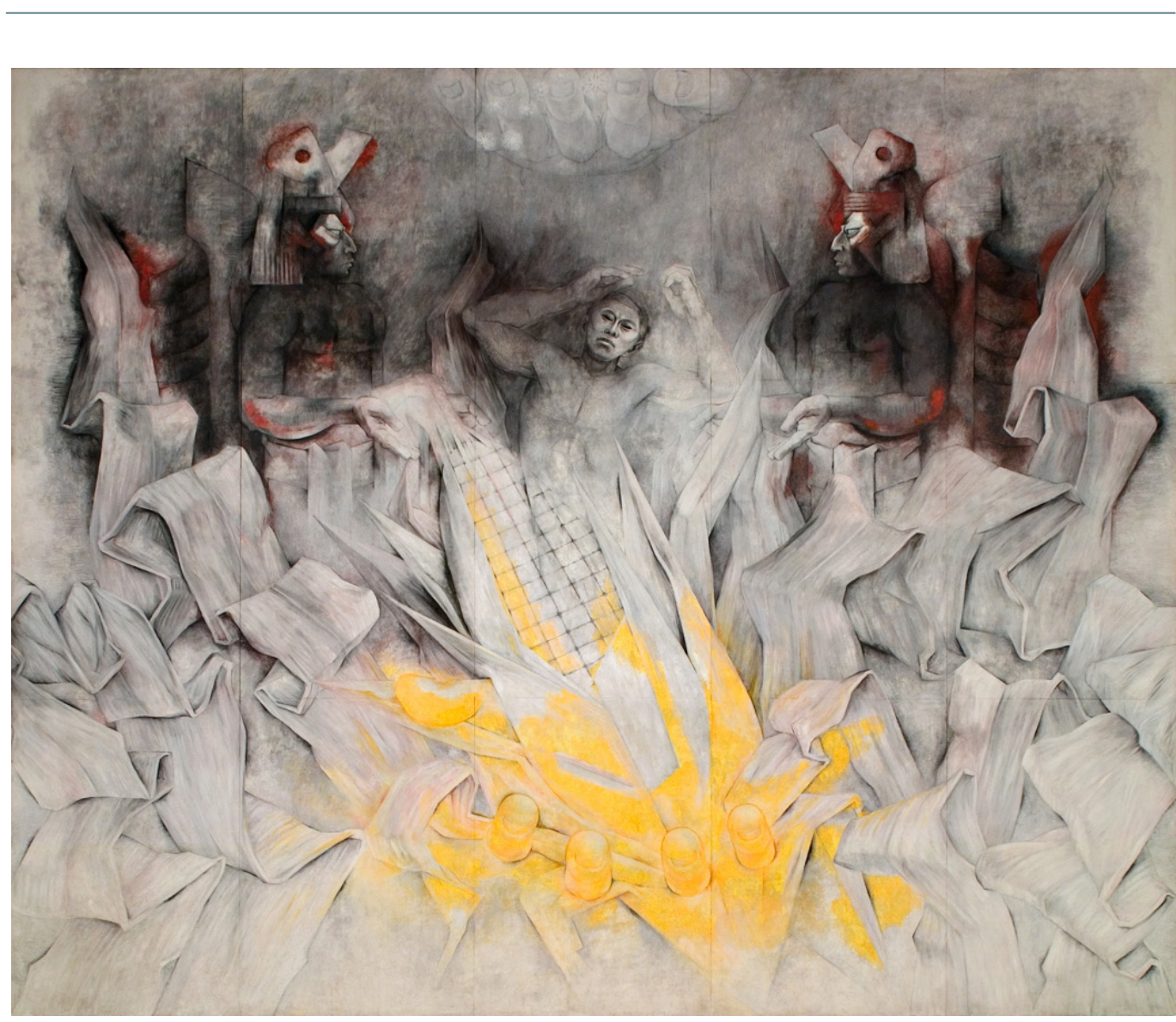

El maíz es, además, la base para la supervivencia de una gran porción de la población de nuestro país. Cerca de 20 millones de habitantes dependen directamente del maíz que producen en sus tierras (sumado al frijol, el chile y la calabaza). La agricultura de subsistencia, aquella de la cual la gente depende directamente, se lleva a cabo en $70 \%$ de las parcelas agrícolas del país. Si bien es cierto que la milpa ocupa un área relativamente pequeña comparada con el cultivo tecnificado, una porción grandísima de la población vive directamente de su tierra. Y la milpa (y el maíz) a su vez depende profundamente del suelo y del clima, en particular de la cantidad y temporalidad de la lluvia.

Para darnos una idea de la profundidad de la interdependencia entre el clima, la producción de alimentos y el bienestar humano, sólo es necesario examinar que pasa con la producción en años particularmente húmedos o secos a nivel nacional. Por ejemplo, los años 1990-

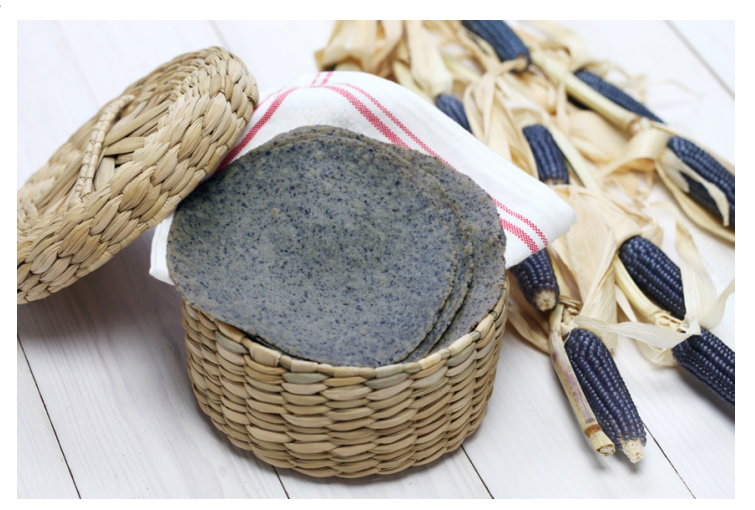


1992 fueron más lluviosos de lo normal en el país, con producciones record de maíz; en dichos años, la migración a Estados Unidos fue la más baja desde 1980. En cambio, durante 1998-1999, un año en el que el fenómeno de El Niño fue particularmente intenso, el país se vio sumergido en una gran sequía. Como era de esperarse, la producción agrícola de maíz de temporal bajó estrepitosamente y la migración hacia nuestro vecino país del norte alcanzó un registro máximo de esa década (algo así como 770 mil personas). En otras palabras, la cantidad de lluvia que cae en nuestro país se traduce directamente en calidad de vida de millones de personas.

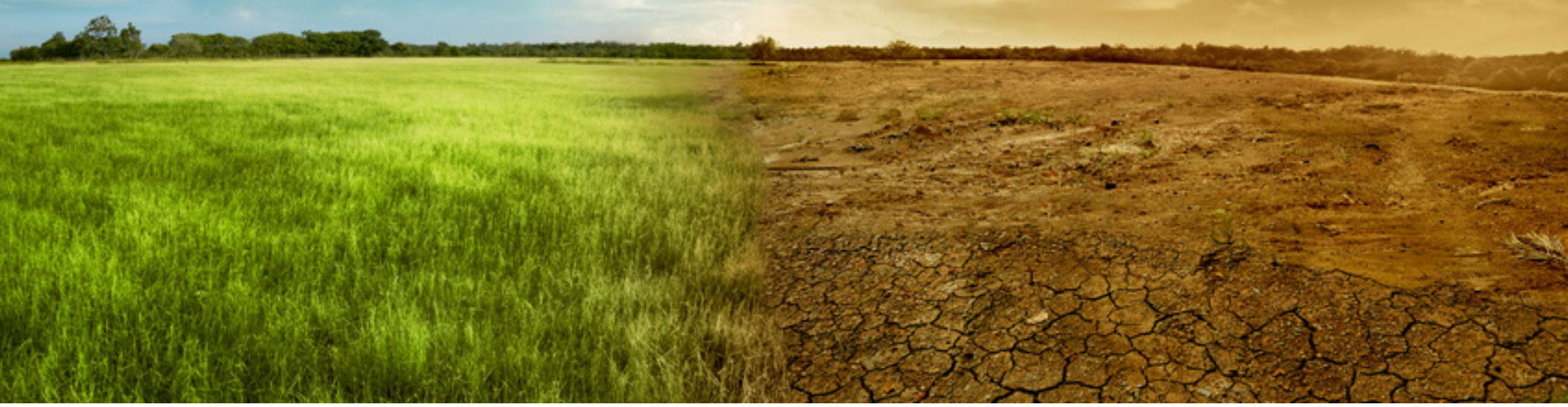

\section{Cambio climático en México: ¿hacia la sequía?}

Aquí es donde se abre el telón y entra el villano más sonado del siglo XXI: el cambio climático global. La quema desmedida de combustibles fósiles que llevamos a cabo, así como la deforestación excesiva para dar paso a potreros y cultivos, han producido un aumento de los gases de efecto invernadero en la atmósfera lo que ha provocado que nuestra Tierra se caliente. Hasta ahora, la temperatura ha aumentado $1^{\circ} \mathrm{C}$ y las predicciones indican que para el 2100 podría ser de entre 2 y $6^{\circ} \mathrm{C}$. Lamentablemente, los datos más recientes muestran que, lejos de frenar nuestra psicótica manera calentar el planeta, cada año aceleramos más la quema de combustibles fósiles. Así lo indican las mediciones de Glen Peters y colaboradores, en un estudio publicado en la revista Nature Climate Change en 2017. 
Lo más preocupante es que con el aumento térmico planetario viene también una redistribución de los patrones de Iluvia. Mayor temperatura equivale a una mayor circulación de los flujos de agua, lo que hace que la precipitación cambie en forma y tiempo. En términos generales, se espera que llueva más en el planeta; sin embargo, se pronostica una disminución en la precipitación o un cambio en su temporalidad regionalmente, particularmente en las zonas áridas. ¿Y cuál es una de esas regiones? Adivinaste bien, es nuestro país.

En los escenarios menos extremos de cambio climático generados por el Panel Intergubernamental de Cambio Climático (el sonado IPCC), la sequía azotaría de manera recurrente al norte y sur de nuestro país, y serían los estados de Nuevo León y Yucatán los más afectados. Pero en los demás escenarios (intermedios o extremos), hacia donde parece que estamos caminando, las Iluvias esquivarían por completo a toda nuestra nación, disminuyendo entre 10 y 15\% a nivel nacional y hasta en 50\% en algunos estados. Para México, el cambio climático equivaldría a una falta de agua para nuestros cultivos y que la lluvia que sí caiga, probablemente lo haga en forma de tormentas intensas o de huracanes.

La sequía, como esta pronosticada, no solo se trata de una disminución en las lluvias, sino también en su temporalidad. Estamos hablando de que el maíz no solo recibirá menos agua mientras crece, sino que posiblemente la duración de la época de lluvias se reduzca, particularmente al inicio. Esto tiene un serio impacto negativo sobre las plántulas de maíz, que en los primeros meses necesitan de la lluvia para poder crecer.

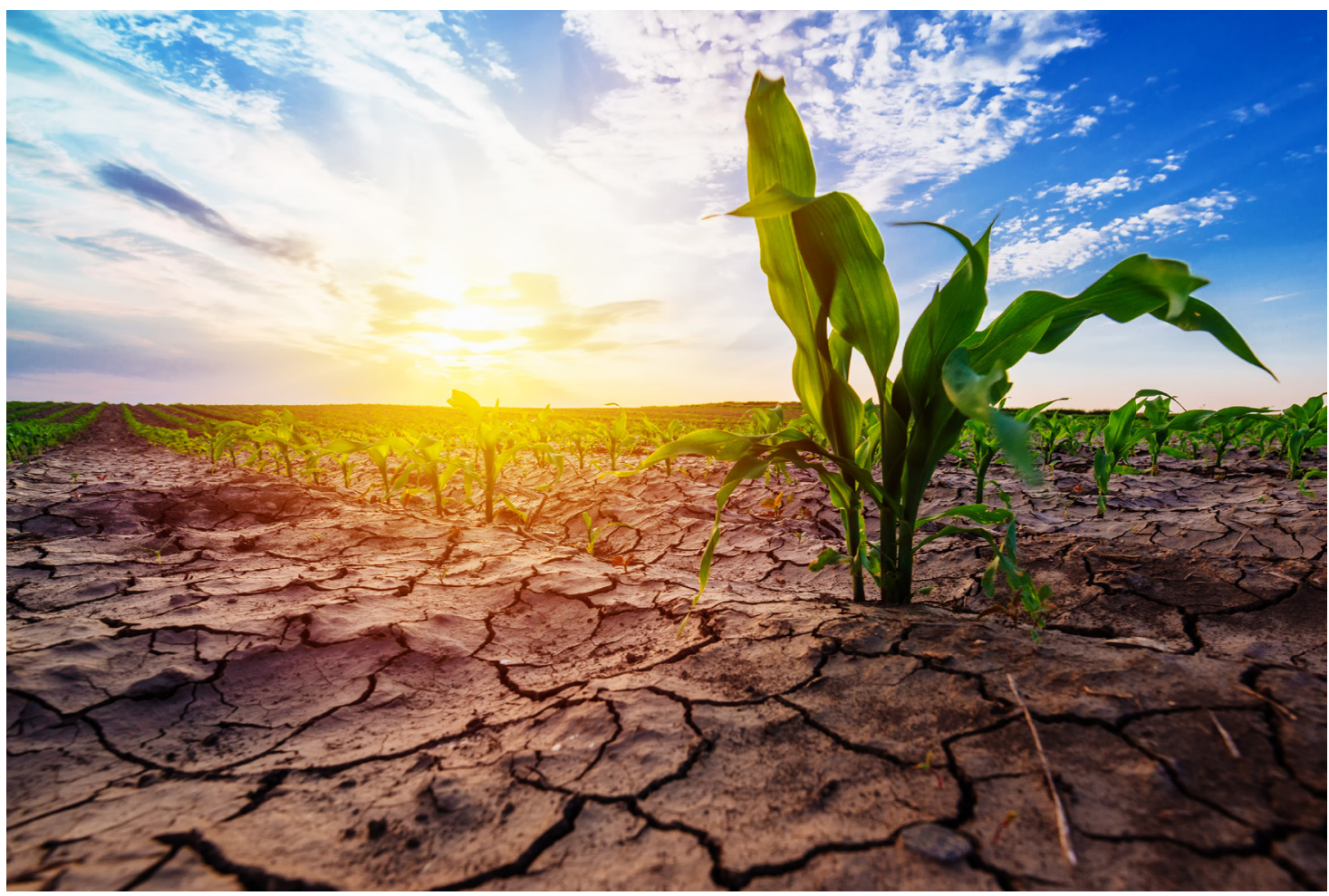


Por supuesto que lo anterior tendría serio impacto para las industrias agrícola y ganadera nacionales. Nuestras estimaciones muestran que la producción nacional de maíz se reduciría hasta en 10\%. Esto afectará particularmente a estados que concentran la agricultura de subsistencia (Oaxaca y Chiapas), donde se pronostica una reducción de hasta 30\% en el rendimiento de las milpas, afectando profundamente a quienes dependen de ella, quienes en general viven en condiciones de pobreza o cercana a ella.

Claramente, el cambio climático en nuestro país tendrá un impacto más grave que el pasar calor en el camión de camino a la escuela o al trabajo, el de no poder dormir en las noches o el no poder usar nuestros suéteres y chamarras en invierno. Hablamos de un impacto potencial directo e indirecto sobre el bienestar de la población, sobre la nutrición de millones de familias y sobre la capacidad para mantenerlas juntas y evitar su migración forzada hacia otros países o del campo a la ciudad. El cambio climático y particularmente la sequía en México, son equivalentes a más pobreza, desnutrición y migración.

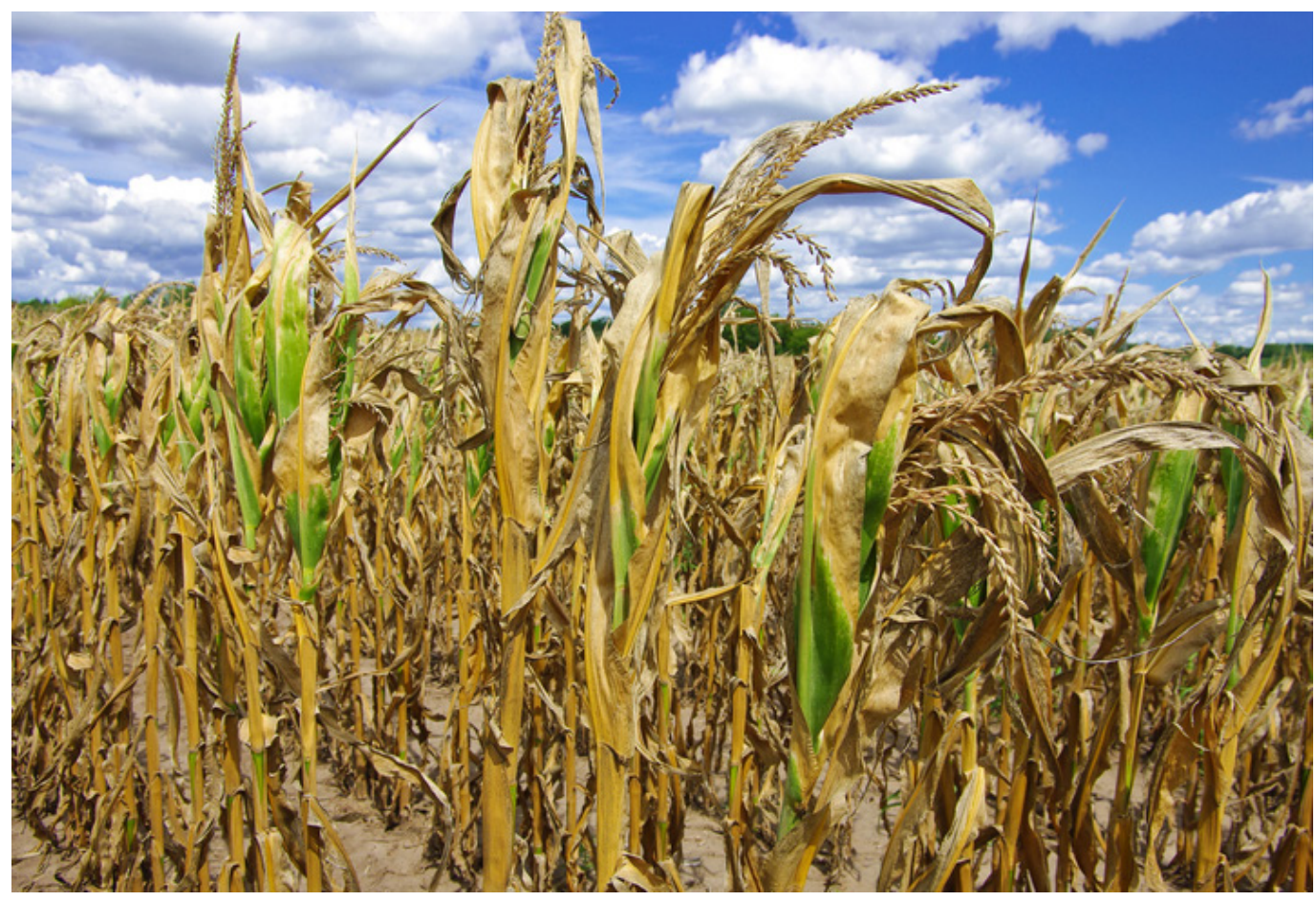

\section{Posibles soluciones: agrotecnología sustentable}

Seguramente, este problema se agudizará con el tiempo, por lo que existe una inminente necesidad de encontrar soluciones para el corto y largo plazos. Aquí mencionamos algunas de ellas, pero hay otras más y se sumarán todas aquellas que todos podemos proponer. En el corto plazo, una de las opciones más viables es el uso de herramientas agrotecnológicas, con una gama de opciones. 


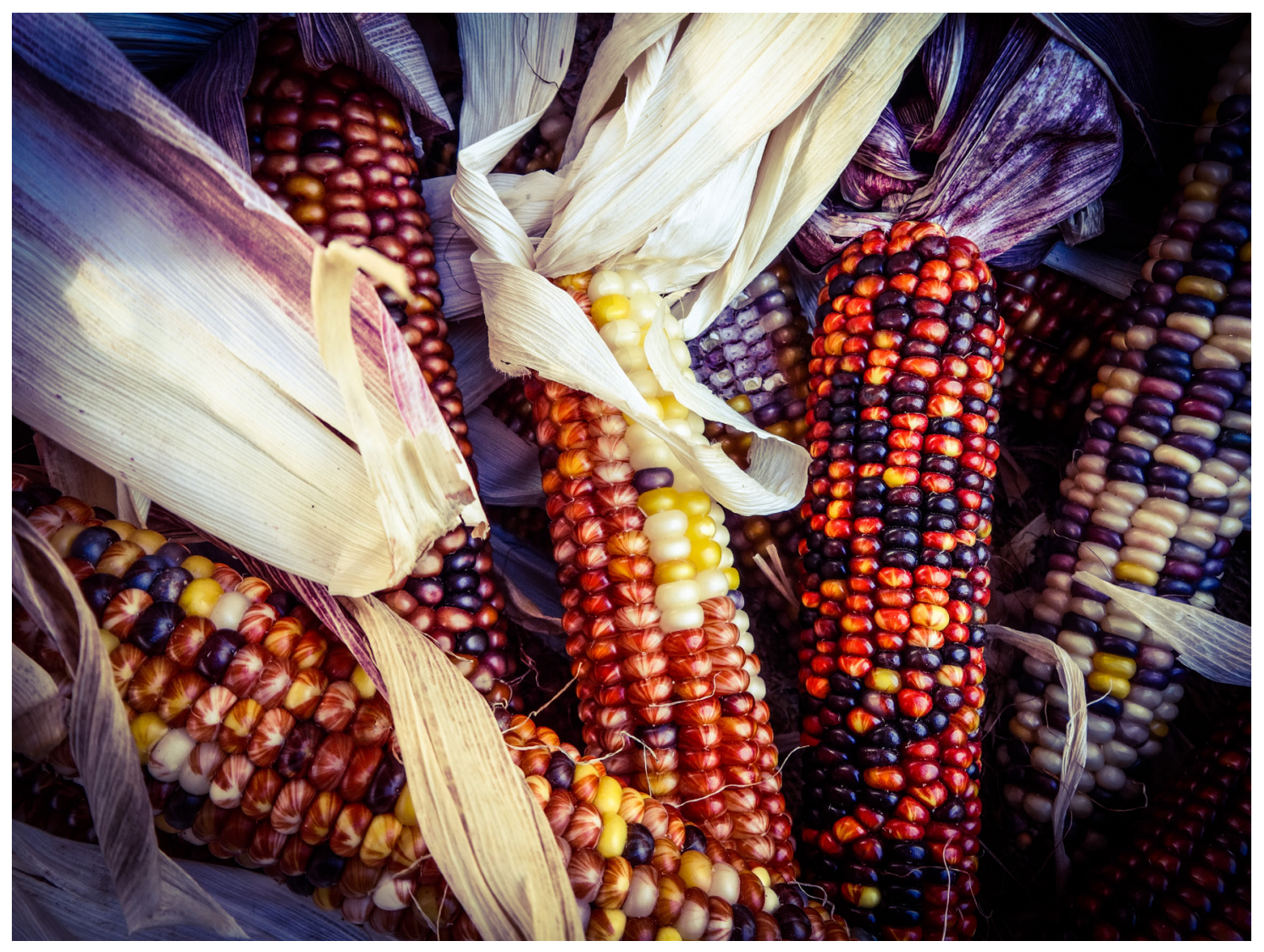

En primer lugar, está la identificación y cultivo de variedades resistentes a la sequía. Esto se ha hecho desde dos perspectivas, la primera, con la recopilación de datos sobre la distribución geográfica de las variedades, como se ha hecho en el Instituto Nacional de Investigaciones Forestales, Agrícolas y Pecuarias (INIFAP). La segunda, mediante herramientas biotecnológicas para la identificación de híbridos en condiciones de laboratorio, como se realiza en el Instituto de Biotecnología de la UNAM y en otras instituciones. La idea es contar con semillas que puedan crecer en condiciones poco favorables de humedad y distribuirlas entre los campesinos que llevan a cabo la agricultura de autosubsistencia. Aquí hay que mencionar, que el proceso de selección de variedades resistentes a la sequía se lleva a cabo de manera tradicional desde hace cerca de 7000 años y es el responsable de la adaptación de 64 razas de maíz a las condicio-

...hay un sinfín de posibles alternativas a los fertilizantes químicos, que representan una inversión muchas veces imposible para los agricultores de pequeña escala y que además tienen serios impactos ambientales. nes locales de México; lo que se busca es acelerar el proceso con los conocimientos de la ciencia moderna.

También se han propuesto otras soluciones más directas. Un cambio en la temporada de siembra es una de ellas; si el maíz se planta más tarde en el año, se limita la vulnerabilidad al riesgo de que se presenten períodos cortos de sequía al inicio de la época de Iluvias. Por ejemplo, Ignacio Sánchez-Cohen 
y colaboradores (2014) del INIFAP encontraron que si los agricultores retrasan un mes la siembra de maíz (en general de mayo a junio) el rendimiento agrícola promedio en Michoacán, Puebla, Jalisco y Oaxaca podría aumentar entre 30 y $300 \%$. Esto gracias a que la época de lluvias es más certera y se disminuye el riesgo de que las plántulas enfrenten condiciones de sequía al inicio de su periodo de crecimiento, crítico para el desarrollo posterior de la planta.

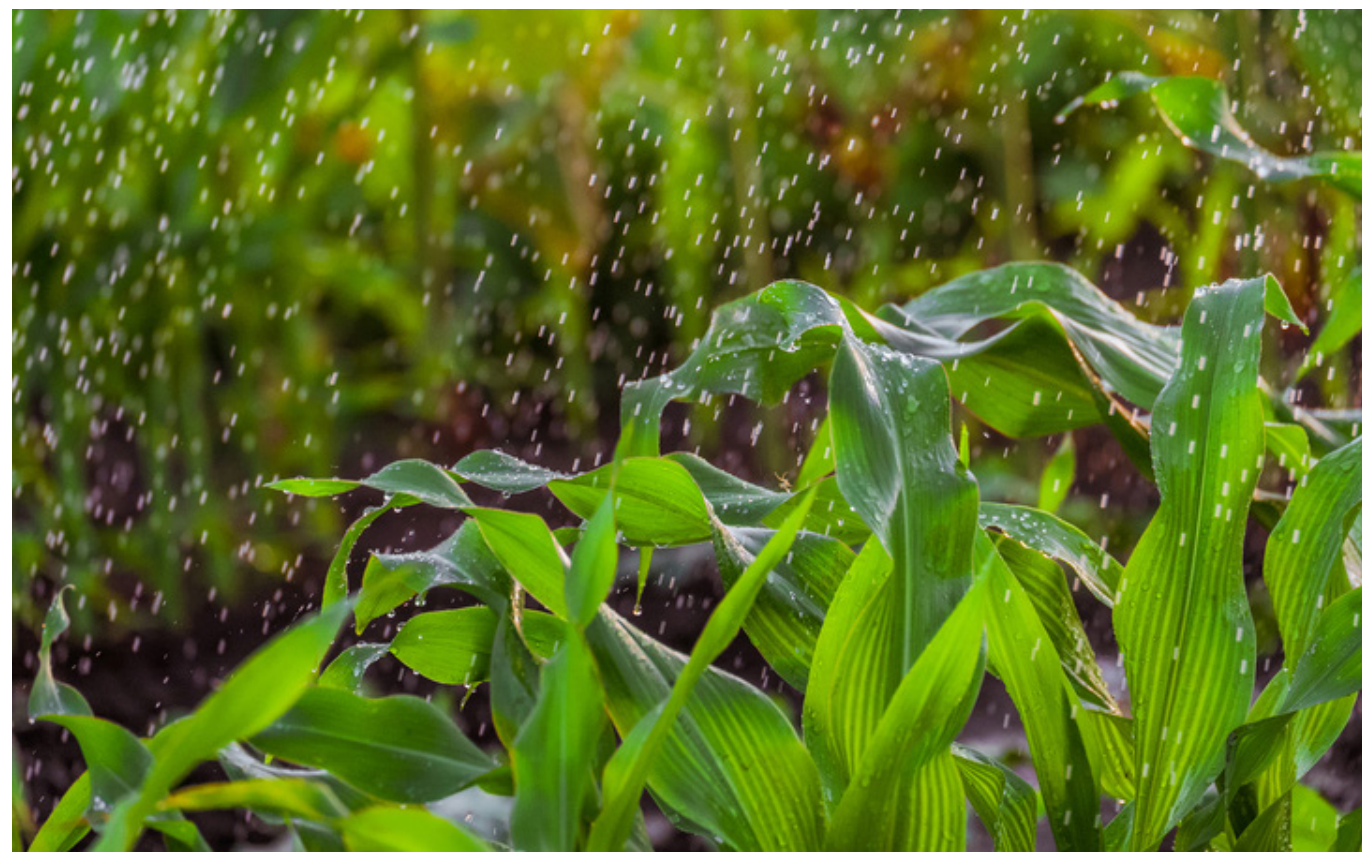

El uso de fertilizantes, que aumentan el rendimiento en las plantas que sobreviven la sequía es otra posibilidad. En este rubro hay un sinfín de posibles alternativas a los fertilizantes químicos, que representan una inversión muchas veces imposible para los agricultores de pequeña escala y que además tienen serios impactos ambientales. Por ejemplo, el uso de fertilizantes orgánicos (como el estiércol), la reutilización de los desechos humanos y los fertilizantes provenientes de algas, lirios y peces -plaga de los lagos del paísrepresentan alternativas para proveer los nutrientes necesarios para el crecimiento del cultivo.

Lo más probable es que se necesite una conjugación de éstas y otras alternativas y el trabajo conjunto de académicos, políticos y campesinos para crear una red de soluciones locales. Proponer soluciones al problema de la producción agrícola frente al cambio climático implica abordar no sólo un asunto económico, sino también ecológico y social. Ahora bien, muchas de estas soluciones estarán en tus manos ¿Tú qué propones? 


\section{Referencias}

* Peters, G. P., Le Quéré, C., Andrew, R. M., Canadell, J. G., Friedlingstein, P., Ilyina, T. y Sitch, S. (2017). Towards real-time verification of $\mathrm{CO}_{2}$ emissions. Nature Climate Change, 1. DOI: https://doi.org/10.1038/s41558-017-0013-9.

- Sanchez Cohen, I., Arriaga, G. E., Valle, M. V., Ibarra, M. A. I., Villalobos, A. M. y Hurtado, P. B. (2014). Climate Based Risk Assessment for Maize Producing Areas in Rainfed Agriculture in Mexico. Journal of Water Resource and Protection, 6(13), 1228. http://dx.doi.org/10.4236/jwarp.2014.613112.

\section{Cómo citar este artículo}

* Contreras Islas, David Sebastián (2017). El reto del Maíz en México frente al cambio climático. Revista Digital Universitaria (RDU). Vol. 19, núm. 1, enero-febrero. DOI: http://doi.org/10.22201/codeic.16076079e.2018.v19n1.a1. 